\title{
IoT in Online Banking
}

\author{
Malti Bansal ${ }^{1, *}$, Naman Oberoi ${ }^{2}$, Mohd. Sameer ${ }^{1}$ \\ ${ }^{1}$ Department of Electronics \& Communication Engineering, \\ Delhi Technological University (DTU), Delhi-110042, India \\ ${ }^{2}$ Department of Mathematics, \\ Delhi Technological University (DTU), Delhi-110042, India
}

maltibansal@gmail.com

\begin{abstract}
As we know, there are so many changes arriving right now ion the banking industries which are really complex industries. Every day, huge amount of data is processed and gathered. With this increase in size, it is becoming more difficult for banking institutions to manage this data and handle other segments of their business. This paper presents the scope of IoT in the banking domain and how various transformations could potentially bring game changing reforms in the traditional methodology. Banking institutions need to integrate IoT in their systems to increase their market share by providing services catered to a clients need based on the data that's being processed in real time. In future, IoT will be able to create such technologies which will be able to connect physical objects so that objects can do their own intelligent decision making.
\end{abstract}

Keywords -Banking, IoT, Big Data, Analytics, Security and Privacy

\section{Introduction}

Banking and financial services serve as the backbone for a country's economy and information technology helps power this infrastructure. For a growing economy, its financial systems should be stable. As the technology advances, the scope of Online Banking increases as it is more convenient for people to do banking online then to go physically in the bank. Like, applying for an account online, doing transactions online, verifying all the details online. All of this provides a major convenience to the users. That's why these days most of the banks are providing online services to the users. Banking involves maintaining relationship with customers and trust if of key nature. Customer satisfaction is key for maintaining lifetime business.

In online banking, a person can perform many processes on his account related to finance using the website of financial institution. We can also call it 'Internet Banking'. As we know, banking systems are where a bank employee deals with the customer and solve his queries physically but in Internet Banking, we are trying to connect the main banking systems with Online Banking to make an ease for the customers.

IoT or Internet of things is a network where there is a transfer and communication of data over the internet through objects which are known as smart devices. It has been widely used in various fields ranging from healthcare, agriculture, smart homes, waste management, etc. There are a total of 5 stages in it. $1^{\text {st }}$ is where we tried to connect 2 systems. When WWW(World Wide Web) is created, many computers can be connected at same time which is the next stage. Then, internet is connected to different devices like mobile phones which are followed by social networking platforms known as people-internet. After that comes the IOT where different objects are connected with each other.

In this paper, we are going to study and discuss things regarding how we can connect IoT with Online Banking. That is how we can do the Online Banking processes with the use of Internet of Things so that we can make some processes easier with it. Rest of the paper is divided into sections as follows. Section II presents what developments in the sector are. Section III describes the position of IoT in the online banking sphere. Section IV highlights the challenges that further need to be addressed and what lies ahead for this industry. Section V gives a conclusive statement to the paper.

\section{When it started and developments done so far}

As far as the online banking domain is concerned, developments are still in a nascent stage with new ideas coming up every day. The existing services can further be enhanced using a standardized framework as proposed by [1]. 
This requires the interpretation of a user's income accounts and balances to provide offers based on the financial condition of the individual and his spending pattern. What further needs to be considered is that such information is secure and not misused by the banks for driving its profitability by using unethical means.

A standard architecture across various papers published consists of the following layers

a) Device management layer - consists of IoT devices that gather data and transmits it to the communication layer. This layer continuously interacts with the physical world. Devices include sensors, telematics from wearables or financial statements of the user.

b) Communication layer - performs functions of the network and transport layer of the traditional internet architecture [1]. This supports routing, error control, handles congestion, QoS, etc. The data is transferred to a cloud platform.

c) Integration layer and analytics layer - This layer helps in storing the information and providing an architecture for analytics on data to take place. This also handles the security of the data.

d) Application layer - this acts as an interface for the user where the user is presented with alerts, offers, reports, etc.

An example of this structure employed is fingerprint authentication. Sensors in device management layer capture the fingerprint and communicate it onto the cloud. Here, it is verified with the data in the database and on successful correspondence, the user is granted access to the banking interface [4].

\section{Where we stand today}

We will now discuss some of the trends that have had a direct impact on the online financial sphere using IoT. In mobile banking, account management has become easier, seamless and can be done instantly and all banking services can be accessed easily. Now, we can access to our bank accounts anywhere anytime. It has become much easier now. To recognise an individual, biometrics is helping a lot because its data is absolutely unique and specific for everyone. Because it uses some advanced techniques. What you need is an account and a platform to access that which is also known as a digital interface.

Removing physical signatures and using 'wet ink technology' is also one of the major achievements. In this, signature of a person is cloned to a paper via a touch screen device so that the need of physical presence of that person is not compulsory.

Monitoring in real time of collaterals and assets is done as a specific identity is provided to the physical objects and hence their tracking and monitoring becomes easier.

Nowadays, everyone wants an instant payment. People are investing in the technologies where physical appearance is not compulsory for transactions. In online banking, you may think there is not enough security but biometrics data is very secure as it is not easy for anyone to access to that data or to understand that data in its original form. So, more and more people are using these online methods now.

There is a platform where you don't need a bank account and you can store and transfer money to other same platforms easily and really fast using 'Wallets of things'. For example, we see PAYTM and PHONEPE apps which have a section known as wallets. Through these sections, we can transfer money to other wallets instantly and in a much easier way.

In future, we can connect an action to a condition. For example, you can take electrical cycles on rent. Suppose you pay rent for $10 \mathrm{~km}$ riding. After you ride $10 \mathrm{~km}$, bike will automatically stop. That is an action (bike stopped) occurred automatically when a condition (rent service finished) arrived.

In Crowd-based financing, small amounts of funds are collected from large amount of crowd using a basic internet platform. In earlier times, you have to go to each house for such payments but now it's all become much easier. 
'PM CARES FUND' is one of the best examples of such financings where funds are collected from a large number of people through online banking methods.

VIRTUAL MONEY is also become really common these days, where you have virtual money in your mobile and you can do payments and transactions through those without having some real money with you.

For securities, biometrics is really enough as these are approximately impossible to access through and even if you get the data, it will be very hard to understand. Like, computers only understand binary languages and it has to convert any other language into binary to actually understand that. So that conversion is nearly impossible for anyone. Also, some venous impressions are created to unable transactions which are impossible to duplicate.

KYC means "Know Your Customer" which is done by bank to get a specific identification of its customer. Some processes are done by bank for KYC to protect their customers from frauds. By using IOT, and giving a specific identity to the customer, special services can be given to special customers through his history. Like, you get much more discount on a shop from where you are buying stuff regularly.

Banks have been quick in terms on investing in new technologies and allotting a significant amount for these services. The number of financial frauds has risen in recent times. The public sector banks have been hit the hardest. With various measures initiated through RBI, number of banking fraud cases have decline, but the amount of money lost has increased [2]. The key piece for the effective implementation of the systems is through employing the best analytics to flag accounts that trigger early warning signals as advised by the RBI.

There has been a significant integration of artificial intelligence for modelling and statistics of data and blockchain for the data privacy. Bluetooth based beacons by Barclays has found use in helping disabled passenger navigate to branches. The use of IoT powered devices such as Alexa, wearables like Samsung gear and fit pay has been crucial in the sense that customers can now pay their bills through it. Banks have started to analyse a customers need through the data obtained, to provide them with sound and smart financial decisions.

\section{Future Scope}

IoT relies on huge data sets to learn about the users and surrounding to better provide interface. However the processing and collection of this data on a huge scale can lead to concerns regarding security and privacy, as well as the management and authentication of a user's identity.

With the amount of data in hand, it also becomes crucial to remove false conclusions from the interpretation of this data which could lead to false positives or false negatives that could label the system as unreliable.

Targeting promotions and ads to users could lead to modifying their behaviour as seen in the Cambridge Analytica scandal. Monitoring and using statistics is what the upcoming models would need to further consider.

Quick response time using analytics on raw data in real time would help in formulating meaningful data from which results can be obtained for the applications. Edge analytics is an area of research to help improve the response time [3].

Banks would further need to employ Big Data in correspondence to IoT to tackle the issues of variety, volume and velocity. Hadoop and MapReduce prove that there is a enormous scope to decrease the detection and/or processing time to prevent fraud [5-13].

Advancements in available hardware and software has led to the development of new methods for the financial service sector. This would lead to a better customer experience, however this is only possible if big data analytics and cloud accessibility are integrated with the IoT structures [1].

\section{Conclusion}

With the integration of IoT in the banking sphere, the strain on banking institutions have reduced as they need to address fewer customers physically.From Table I. [3], India remains a largely untapped market in terms of banking online with only $48 \%$ of the population engaged. IoT can help capture this untapped market and for its successful 
implementation, banking institutions would need to strive to make a uniform customer interface that is easy for the general public to use. Future banking will circle around IoT being the critical support for the institutions products and services. It will lead to essential developments in the banking sectors and hence we will see more ease in money transactions, creating bank accounts, security of our accounts, etc. As the graph of development in IOTs is exponential, we will see these changes really quickly and we will be able to observe those in our day-today lives.

\section{References}

[1] Vemula, Dr-Dinesh Reddy \& Gangadharan, G R. (2016). Towards an Internet of Things Framework for Financial Services Sector. 10.1109/RAIT.2016.7507897.

[2] R. S. Lande, S. A. Meshram and P. P. Deshmukh, "Smart banking using IoT," 2018 International Conference on Research in Intelligent and Computing in Engineering (RICE), San Salvador, 2018, pp. 1-4, doi: 10.1109/RICE.2018.8627903.

[3] H. Ramalingam and V. P. Venkatesan, "Conceptual analysis of Internet of Things use cases in Banking domain," TENCON 2019 - 2019 IEEE Region 10 Conference (TENCON), Kochi, India, 2019, pp. 2034-2039, doi: 10.1109/TENCON.2019.8929473.

[4] A. Taralekar, G. Chouhan, R. Tangade and N. Shardoor, "One touch multi-banking transaction ATM system using biometric and GSM authentication," 2017 International Conference on Big Data, IoT and Data Science (BID), Pune, 2017, pp. 60-64, doi: 10.1109/BID.2017.8336574.

[5] Boumlik, Abdeljalil \& Bahaj, Mohamed. (2018). Big Data and IoT: A Prime Opportunity for Banking Industry. Lecture Notes in Networks and Systems. 396-407. 10.1007/978-3-319-69137-4_35.

[6] Du, X., Zhou, Z., Zhang, Y. et al. Energy-efficient sensory data gathering based on compressed sensing in IoT networks. J Cloud Comp 9, 19 (2020). https://doi.org/10.1186/s13677-020-00166-X

[7] A. Al-Fuqaha, M. Guizani, M. Mohammadi, M. Aledhari and M. Ayyash, "Internet of Things: A Survey on Enabling Technologies, Protocols, and Applications," in IEEE Communications Surveys \& Tutorials, vol. 17, no. 4, pp. 2347-2376, Fourthquarter 2015, doi: 10.1109/COMST.2015.2444095.

[8] Fadoua Khanboubi, Azedine Boulmakoul, Mohamed Tabaa,Impact of digital trends using IoT on banking processes,Procedia Computer Science,Volume 151,2019,Pages 77-84,ISSN 1877-0509, https://doi.org/10.1016/j.procs.2019.04.014.(http://www.sciencedirect.com/science/article/pii/S1877050919304 $\underline{752)}$

[9] M. Bansal and Priya, "Application Layer Protocols for Internet of Healthcare Things (IoHT)," 2020 Fourth International Conference on Inventive Systems and Control (ICISC), Coimbatore, India, 2020, pp. 369-376, doi: 10.1109/ICISC47916.2020.9171092.

[10] M. Bansal, Priya, "Performance Comparison of MQTT and CoAP Protocols in Different Simulation Environments". G. Ranganathan, J. Chen, A. Rocha (eds) Inventive Communication and Computational Technologies. Lecture Notes in Networks and Systems, vol. 145, pp. 549-560, Springer, Singapore, https://doi.org/10.1007/978-981-15-7345-3 47

[11] M.Bansal, Priya, "Machine Learning Perspective in VLSI Computer Aided Design at Different Abstraction Levels", $2^{\text {nd }}$ Int. Conf. on Mobile Computing and Sustainable Informatics (ICMCSI 2021), Tribhuvan University, Nepal, 2021.

[12] M.Bansal, A. Goyal, A. Choudhary, "Industrial Internet of Things (IIoT): A Vivid Perspective", $5^{\text {th }}$ Int. Conf. on Inventive Systems and Control (ICISC 2021), India, 2021.

[13] M. Bansal, V. Sirpal, M. K. Choudhary, “Advancing e-Government using Internet of Things", ”, $2^{\text {nd }}$ Int. Conf. on Mobile Computing and Sustainable Informatics (ICMCSI 2021), Tribhuvan University, Nepal, 2021. 